\section{International Scientific Journal Theoretical \& Applied Science}

p-ISSN: 2308-4944 (print) e-ISSN: 2409-0085 (online)

Year: $2014 \quad$ Issue: $10 \quad$ Volume: 18

Published: $30.10 .2014 \quad$ http://www.T-Science.org
Nina Aleksandrovna Kazachek junior research scientist, assistant of the department "Problems of control"

Moscow State Technical University of Radio Engineering, Electronics and Automation (MSTU MIREA), Russia nkazachek@yandex.ru

Sergey Viktorovich Yepishin Programmer, MSTU MIREA, Russia

SECTION 4. Computer science, computer engineering and automation.

\author{
Maksim Sergeevich Melikhov
}

Vladimir Andreevich Ryabcov

Student, MSTU MIREA, Russia

\title{
IMPROVING THE PERFORMANCE OF CONTROL AND GUIDANCE SYSTEMS BASED ON FUZZY CONTROLLERS
}

Abstract: In this paper, a method for increasing control and guidance system performance through the use of the controller implemented on the basis of fuzzy inference is proposed. Comparative analysis of the servo system with the classical PID controller and fuzzy P controller is given.

Key words: PID - controller, fuzzy inference, fuzzy P controller, the error in the steady state, the setting time

Citation: Kazachek NA, Yepishin SV, Melikhov MS, Ryabcov VA (2014) IMPROVING THE PERFORMANCE OF CONTROL AND GUIDANCE SYSTEMS BASED ON FUZZY CONTROLLERS. ISJ Theoretical \& Applied Science 10 (18): 1-6. doi: http://dx.doi.org/10.15863/TAS.2014.10.18.1

\section{ПОВЫШЕНИЕ КАЧЕСТВА СИСТЕМ УПРАВЛЕНИЯ И НАВЕДЕНИЯ НА ОСНОВЕ НЕЧЕТКИХ РЕГУЛЯТОРОВ}

Аннотация: В данной работе предлагается метод повышения качества функционирования систем управления и наведения, путем использования регулятора, реализованного на базе нечеткого логического вывода. Приводится сравнительный анализ работы следящей системы с классическим ПИД- регулятором и нечетким П-регулятором.

Ключевые слова: ПИД - регулятор, нечеткий логический вывод, нечеткий П-регулятор, ошибка в установившемся режиме, время регулирования

\section{Введение}

Современный уровень развития техники специального назначения требует новых подходов к разработке регуляторов и систем автоматического управления данными объектами. Это обусловлено, с одной стороны, необходимостью повышения качества управления при минимальных затратах на создание и эксплуатацию систем, с другой стороны усложнением структуры объекта управления, функций, выполняемых им, и, как следствие, увеличением факторов неопределённости, которые необходимо учитывать для управления объектом $[8,9]$.

Известные методы адаптивного управления в быстродействующих системах как гражданского, так и специального назначения, применения не нашли. Связано это как со сложностью адаптивных алгоритмов, которые требуют больших вычислительных мощностей, приводящих к появлению запаздывания в контуре системы и к соответствующему ухудшению ее динамики, так и трудностями их реализации на цифровой технике с учетом условий обеспечения устойчивости дискретной системы управления. При этом можно и нужно говорить о реальности применения существующей элементной базы для создания определенных классов интеллектуальных систем управления, относительная простота которых связана с обработкой ограниченного набора знаний в конкретной предметной области. Кроме того, уже существует фундаментальная теоретическая база (работы Д. А. Поспелова, Л. Заде и других ученых $[1,2])$, используя результаты которой в сочетании с пониманием теории управления могут принести свои плоды в обоснованной интеллектуализации 
систем автоматического управления [3]. Таким образом, перспективы развития технологий интеллектуального управления абсолютно прозрачны и ясны, и кроме того, они связаны с возможностью обеспечения высоких показателей точности, быстродействия и других характеристик в сочетании с инвариантностью к возмущающим факторам [4, 6, 7, 10]. Технология нечеткой логики [5] находит все более широкое применение для создания интеллектуальных регуляторов в системах автоматического управления современными образцами гражданской и специальной техники различных типов и назначения.

Сравнительный анализ работы следящей системы с классическим пид- регулятором и нечетким I - регулятором
В данной работе проводится оценка качественных показателей следящей системы автоматического управления с классическим ПИД - регулятором и нечетким П- регулятором. В роли исследуемого объекта возьмем систему регулирования скорости движения ракетыносителя, представленную на рисунке 1.

Для данной системы настроим регуляторы: классический ПИД - регулятор по методу ЗиглераНикольса (Кп=12, Ки=3, Кд=0,00005); нечеткий Прегулятор типа 1, обеспечивающий апериодический переходной процесс, вид нелинейности представлен на рисунке 2 ; нечеткий регулятор типа 2, обеспечивающий компенсацию внешних возмущений, рисунок 3 .

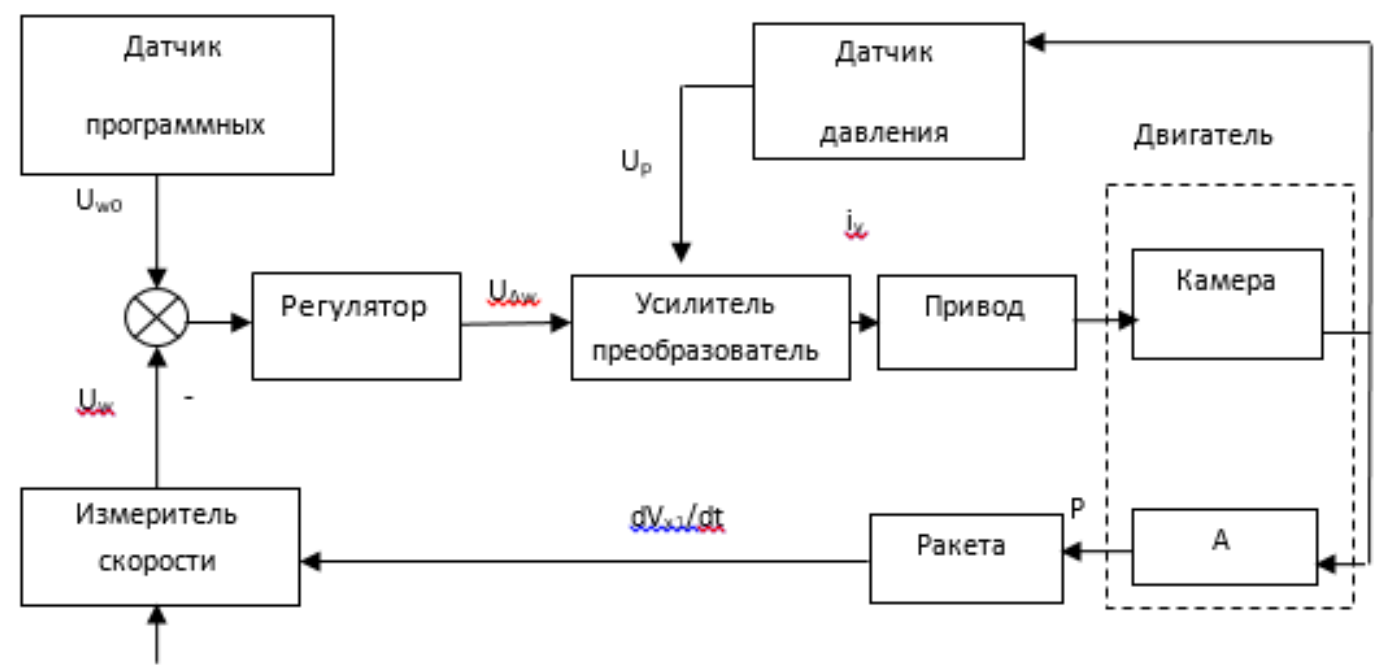

Рисунок 1 - Система регулирования скорости движения ракеты-носителя.

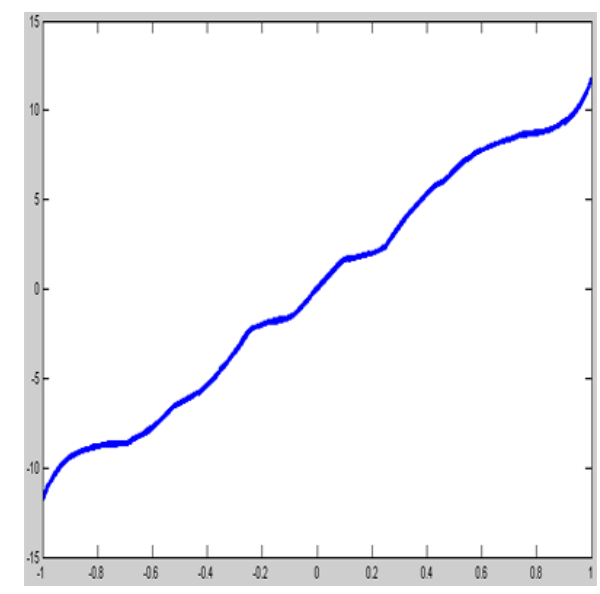

Рисунок 2 - Нелинейность, реализуемая нечетким П-регулятором типа 1. 


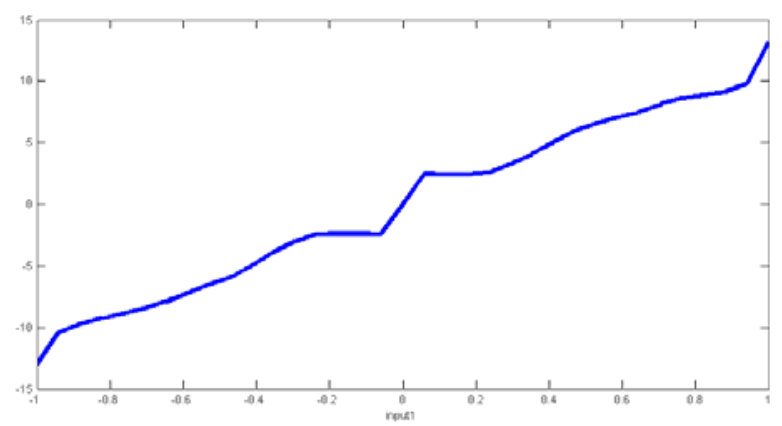

Рисунок 3 - Нелинейность, реализуемая нечетким П -регулятором типа 2.

Произведем сравнение классического ПИДрегулятора и нечеткого П-регулятора типа 1. Сначала оценим качество работы систем при отсутствии внешних возмущений в системе. Подадим на вход систем константы различной величины, чтобы оценить качество работы регуляторов. Как видно из результатов (рисунок 4), ПИД-регулятор, настроенный по методу ЗиглераНикольса, при отсутствии внешних возмущений в системе создает перерегулирование порядка 7-8\%,a также проигрывает по времени регулирования.

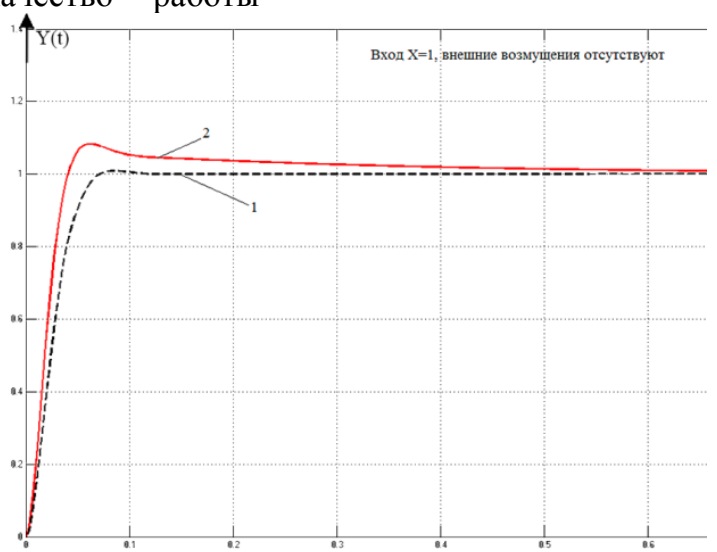

Рисунок 4 - Переходные процессы систем с П-нечётким типа 1 (1) и классическим ПИДрегулятором(2) при подаче входного сигнала $X=1$ и отсутствии внешнего возмущения.

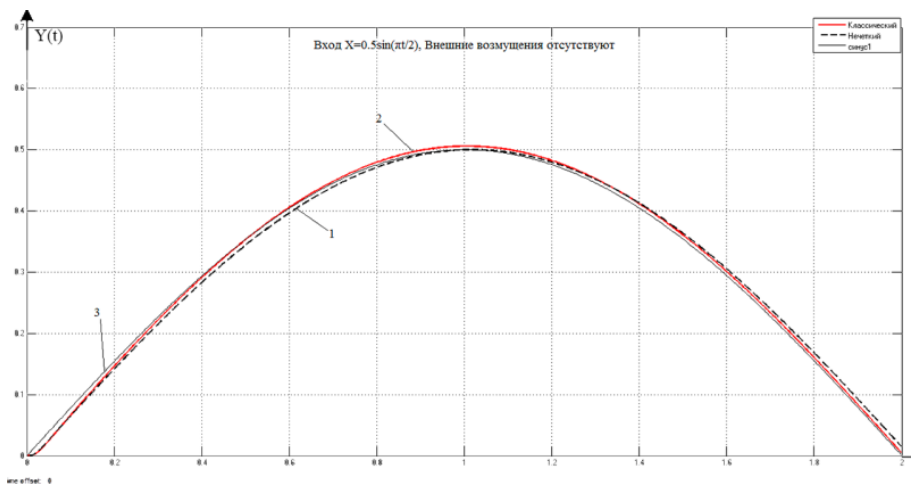

Рисунок 5 - Переходные процессы систем с П-нечётким типа 1 и классическим ПИДрегулятором(2), входное значение $X=0.5 \sin (\pi t / 2)(3)$ и отсутствие внешних возмущений.

Если подать на вход системы синусоидальную функцию,то показатели качества обоих систем будут практически идентичны, рисунок 5:

Однако при наличие в системе внешнего возмущения отличного от 0 , нечеткий П-регулятор типа 1 оставляет ошибку в установившемся режиме (до $3 \%$ ), что легко объяснимо отсутствием интегральной составляющей в данном регуляторе.
Так как основным достоинством рассчитанного ПИД-регулятора оказалась способность нейтрализовать внешние возмущенияконстанты, целесообразно сравнить его с нечетким П-регулятором типа 2, настроенным на нейтрализацию внешних возмущений.

Сначала сравним качество систем при отработке входных заданий без внешних возмущений. По результатам моделирования 
(рисунок 6) можно сделать выводы, что в системе с нечетким П-регулятором типа 2, перерегулирование меньше, время регулирования немного больше, чем в системе с классическим ПИД-регулятором.
При отработке входных сигналов синусоидального характера, система с нечетким Прегулятором типа 2 показала гораздо более высокую точность, чем система с ПИДрегулятором, рисунок 7.
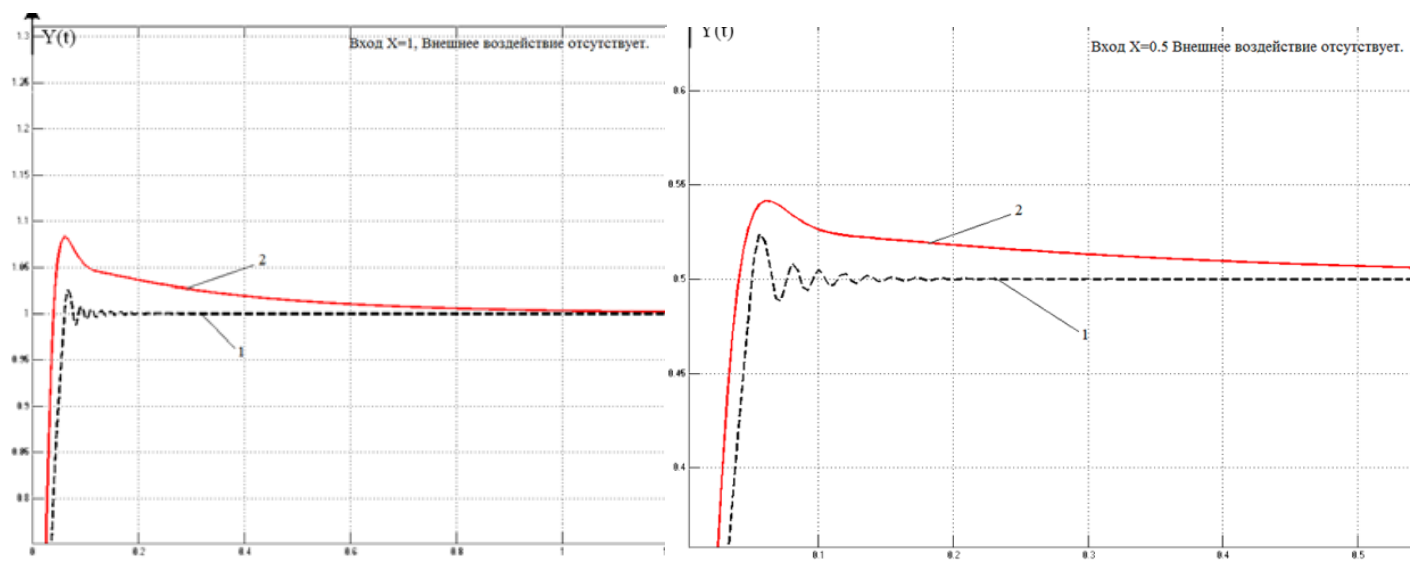

Рисунок 6 - Переходные процессы систем с П-нечётким типа 2 (1) и классическим ПИДрегулятором(2), входной сигнал $X=1 / 0,5$ и отсутствие внешних возмущений.

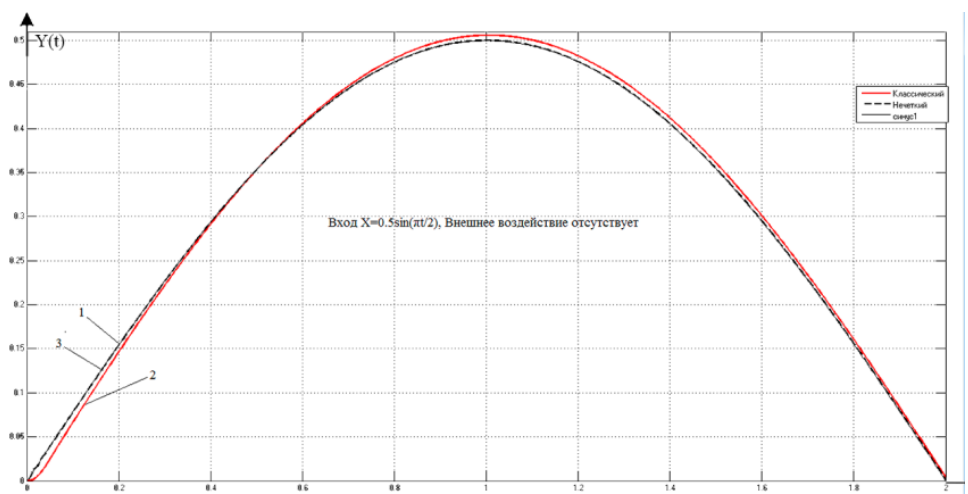

Рисунок 7 - Переходные процессы систем с П-нечётким типа 2(1) и классическим ПИДрегулятором(2), входной сигнал $X=0.5 \sin (\pi t / 2)(3)$.

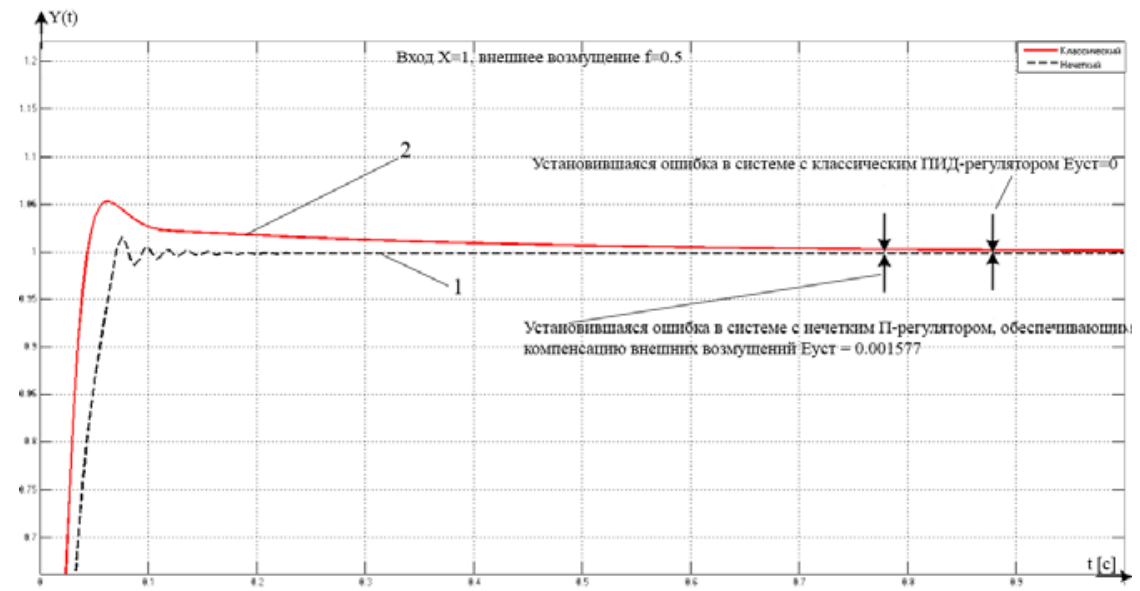

Рисунок 8 - Переходные процессы систем с П-нечётким типа 2 (1) и классическим ПИДрегулятором(2), входной сигнал $X=1$, и внешнее возмущение $\mathbf{f}=0.5$. 
Система с нечетким П-регулятором типа 2 обеспечивает высокие показатели качества в установившемся режиме - при внешнем возмущении-константе $\mathrm{f}=1$, отклонение от требуемого значения составляет всего лишь $0,48 \%$, т.е. установившаяся ошибка стремится к нулю.

Полученный результат объясняется тем, что у нечеткого П-регулятора типа 2, настроенного на компенсацию внешних возмущений, угол наклона нелинейности вблизи нуля высок, а значит - на нейтрализацию ошибки идет гораздо больший выход регулятора, чем в случае нечеткого Прегулятора типа 1 с апериодическим переходным процессом. Это сделано для максимально эффективной компенсации установившейся ошибки, не сильно «поломав» при этом переходной процесс. Данный регулятор можно использовать, если требования к системе допускают наличие незначительного перерегулирования, но гораздо важнее компенсировать возмущения, действующие на систему.

В случае же синусоидальных внешних воздействий (рисунок 9) очевидно превосходство нечеткого П-регулятора типа 2 в компенсации таких возмущений. При синусоидальном возмущении $\mathrm{f}=\sin (\pi \mathrm{t})$, выход системы с нечетким регулятором колеблется с амплитудой 0.003 , в то время как в системе с классическим ПИДрегулятором, колебания происходят с амплитудой 0.0485 .

В случае, если целью управления является компенсация возмущений - данный нечеткий Прегулятор типа 2 вполне может заменить классический ПИД-регулятор.

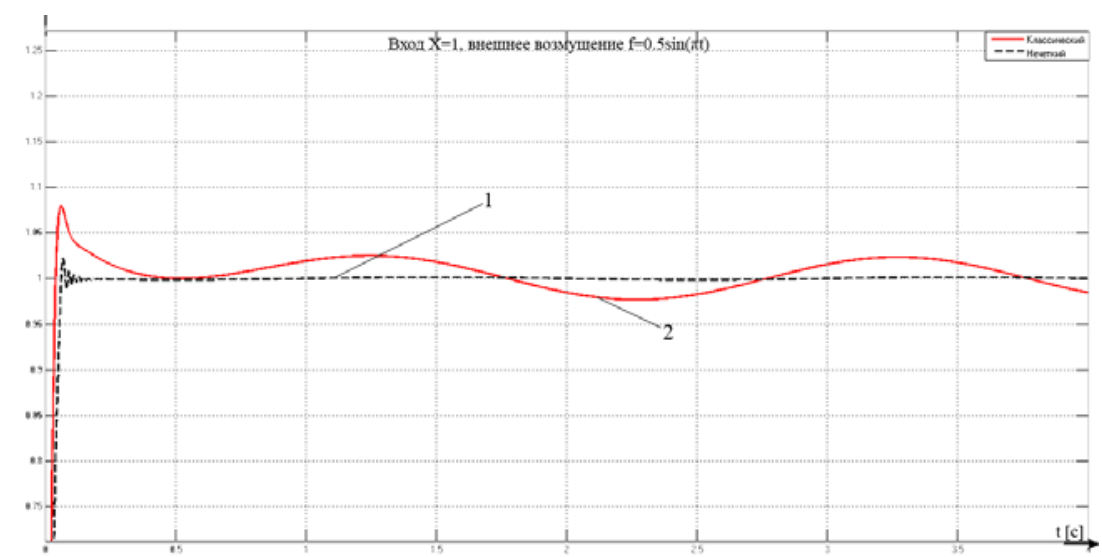

Рисунок 9 - Переходные процессы систем с П-нечётким типа 2 (1) и классическим ПИД-регулятором(2), входной сигнал $X=1$ и внешнее возмущение $f=0.5 \sin (\pi t)$.

\section{Заключение}

Подведем итог проведенных исследований. В зависимости от требований к системе автоматического управления, нечеткий Прегулятор может решить ту или иную задачу, для решения которой, как правило, применяют классический ПИД-регулятор: обеспечение оптимального быстродействия системы, апериодического переходного процесса, компенсации внешних возмущений, отработки входных сигналов, являющихся линейными функциями от времени. При этом, в большинстве своем, система с нечетким П-регулятором обеспечивает эквивалентные системе с классическим ПИД -регулятором показатели качества, а в ряде случаев значительно превосходит их. Объясняется это однородностью кривой управления ПИД-регулятора на всех участках, в то время как с помощью нечеткой логики можно задавать форму кривой индивидуально на каждом ее участке, максимально нивелировав действие каждого из участка на соседние.

\section{References:}

1. Metody issledovaniya nelineynykh sistem avtomaticheskogo upravleniya (1975) Pod red. RA Nelepina. Moscow, Nauka.

2. Makapov IM, Lokhin VM, Man'ko SV, Romanov MP, Sitnikov MS (2013) Ustoychivost' intellektual'nykh sistem avtomaticheskogo upravleniya (uchebnoe posobie s grifom UMO). Informatsionnye tekhnologii. Prilozhenie. No. 2.

3. Intellektual'nye sistemy avtomaticheskogo upravleniya (2001) Pod red. IM Makarova, VM Lokhina. Moscow, Fizmatlit, 576.

4. Makarov IM, Lokhin VM, Man'ko SV, Romanov MP, etc (2006) Iskusstvennyy intellekt i 
intellektual'nye sistemy upravleniya. Moscow: Nauka, 333.

5. Pegat A (2013) Nechetkoe modelirovanie i upravlenie. per. s angl., 2 izd. Moscow: BINOM, 798.

6. Makarov IM, Lokhin VM, Man'ko SV, Romanov MP, etc (2009) Avtomatizatsiya sinteza i obuchenie intellektual'nykh sistem upravleniya (monografiya). Moscow: Nauka, 228.

7. Makarov IM, Lokhin VM, Man'ko SV, Romanov MP (2006) Iskusstvennyy intellekt i intellektual'nye sistemy upravleniya (monografiya). Moscow: Nauka.

8. Makarov IM, Lokhin VM, Man'ko SV, Romanov MP, Ivlev AA, Yurin AD (2009) Perspektivy i realii primeneniya intellektual'nykh tekhnologiy upravleniya i obrabotki informatsii pri sozdanii obraztsov vooruzheniya i voennoy tekhniki. Mekhatronika, avtomatiza-tsiya, upravlenie No.3.

9. Makarov IM, Lokhin VM, Man'ko SV, Romanov MP, Aleksandrova RI (2013) Razvitie tekhnologii intellektual'nogo upravleniya dlya sozdaniya perspektivnykh obraztsov VVT na baze novykh sredstv kompleksnoy avtomatizatsii proektirovaniya. Izvestiya YuFU. Tekhnicheskie nauki No.3.

10. Makarov IM, Lokhin VM, Man'ko SV, Romanov MP, Sitnikov MS (2009) Dinamika intellektual'nykh $\mathrm{V}$ malom sistem avtomaticheskogo upravleniya. Trudy XVIII Mezhdunarodnogo nauchno-tekhnicheskogo seminara. «Sovremennye tekhnologii v zadachakh upravleniya, avtomatiki i obrabotki informatsii». Moscow.: MIREA. 